FTUV-01/1001 IFIC-00-67

DAMTP-2000-136

January 9, 2001

\title{
Hidden supersymmetries in supersymmetric quantum mechanics
}

\author{
J. A. de Azcárraga ${ }^{a 1}$, J. M. Izquierdo ${ }^{b 1}$ and A. J. Macfarlane㝏 \\ a Departamento de Física Teórica, Universidad de Valencia \\ and IFIC, Centro Mixto Universidad de Valencia-CSIC, \\ E-46100 Burjassot (Valencia), Spain \\ b Departamento de Física Teórica, Universidad de Valladolid \\ E-47011 Valladolid, Spain \\ c Centre for Mathematical Sciences, D.A.M.T.P. \\ Wilberforce Road, Cambridge CB3 OWA, UK
}

\begin{abstract}
We discuss the appearance of additional, hidden supersymmetries for simple $0+1 A d(G)$-invariant supersymmetric models and analyse some geometrical mechanisms that lead to them. It is shown that their existence depends crucially on the availability of odd order invariant skewsymmetric tensors on the (generic) compact Lie algebra $\mathcal{G}$, and hence on the cohomology properties of the Lie algebra considered.
\end{abstract}

\footnotetext{
${ }^{1}$ j.a.de.azcarraga@ific.uv.es, izquierd@fta.uva.es, a.j.macfarlane@damtp.cam.ac.uk
} 


\section{Introduction}

In supersymmetric quantum mechanics models with standard supersymmetry, the supercharges $Q_{a}$ are related to the Hamiltonian $H$ via $\left\{Q_{a}, Q_{b}\right\}=$ $H \delta_{a b}, a, b=1, \ldots, N$. In many of these models one can find additional or 'hidden' supercharges $\tilde{Q}$ [1, 2], involving the structure constants of a Lie algebra, and perhaps a Killing-Yano tensor [3, 田. The appearance of the Killing-Yano tensor in this context is not surprising, since it also plays a role in the existence of hidden symmetries [5, 6].

The additional supercharges are required to satisfy

$$
\left\{Q_{a}, \tilde{Q}\right\}=0
$$

hence $[\tilde{Q}, H]=0$, so that the $\tilde{Q}$ 's generate supersymmetries of the theory. We shall consider three models: one with bosonic superfields, and two with fermionic superfields (with $N=1$ and $N=2$ respectively) for which the bosonic component variables are auxiliary [7].

A typical example of the bosonic superfield case, in which the Lie algebra $\mathcal{G}$ is $s u(2)$, is that of the non-relativistic motion of a spin- $\frac{1}{2}$ particle in the background field of a Dirac monopole [8] (see also [9]). The case $G=S U(2)$ is, however, rather exceptional since it is the only group for which the structure constants of its algebra coincide with the fully antisymmetric tensor $\left(\epsilon_{i j k}\right)$ of a $(\operatorname{dim} G)$-dimensional space. Thus, a natural question to ask is what generalisations are possible when simple (and compact) algebras $\mathcal{G}$ of rank $l>1$ are employed. Also, for $l>1$ there exist other available skewsymmetric tensors (of odd order $>3$ ): they are provided by the higher order cocycles of the Lie algebra cohomology of $\mathcal{G}$. Thus, in order to investigate the appearance of hidden charges in a group theoretical context, we look first at

$$
\tilde{Q}_{3}=\dot{x}_{i} f_{i j} \psi_{j}-\frac{1}{3} i f_{i j k} \psi_{i} \psi_{j} \psi_{k} \quad,
$$

where $x_{i}$ and $\psi_{i}$ are the position and fermionic coordinates respectively, $i=$ $1, \ldots, \operatorname{dim} \mathcal{G}$, and $f_{i j}$ is an antisymmetric second order Killing-Yano tensor [3, [] associated with the structure constants $f_{i j k}$ in such a way that (11) holds, and second we look at

$$
\tilde{Q}_{5}=\dot{x}_{i} f_{i j k l} \psi_{j} \psi_{k} \psi_{l}-\frac{1}{5} i \Omega_{i j k p q} \psi_{i} \psi_{j} \psi_{k} \psi_{p} \psi_{q}
$$


in various contexts. In (3),$f_{i j k l}=f_{[i j k l]}$ is a fourth order generalised KillingYano tensor and $\Omega_{i j k p q}$ is a fifth order totally antisymmetric invariant tensor, associated with the third order (Racah-Casimir) invariant symmetric polynomial of such $\mathcal{G}$ as allow for one. This exists for $s u(n), n \geq 3$, and $s u(3)$ will be good enough to illustrate the extent of most of our results when using the fifth order cocycle.

In the fermionic superfield case, it is possible to construct models where the only dynamical fields are fermionic, and whose Lagrangian includes an interaction term constructed using the structure constants of the simple Lie algebra. Our aim is to construct, in terms of the corresponding fermionic variables and the higher order cocycles, hidden supercharges in the $N=1$ and $N=2$ cases. When $N=1$ we shall restrict the Lie algebra to $s u(n)$, whereas in the $N=2$ case the simple Lie algebra $\mathcal{G}$ will be unrestricted. The restriction reflects the fact that the discussion of the $N=1$ case employs the identity $C_{i j k} \Omega_{i j k s_{4} \ldots s_{2 m-1}}=0$, which we believe holds for all $\mathcal{G}$, but for which explicit detailed proofs are available [10] only for $s u(n)$.

We consider only $A d(G)$-invariant simple $0+1$ supersymmetry models. In Sec. 2 we describe the case of a non-relativistic system moving in a space that is the representation space of the adjoint representation of the symmetry group $G$, coupled to a background potential $A_{i}$. It is shown in sec. 3 that in the free case there exist non-standard supersymmetries associated with all the higher order cocycles. In the presence of the background field $A_{i}$ (sec. 4 ), however, we find only one hidden supercharge for the lowest order cocycle i.e., for that given by the structure constants of $\mathcal{G}$.

In Sec. 5, we consider a $N=1$ simple purely fermionic model and show that one may also construct hidden supercharges from each of the $l s u(n)$ algebra cohomology cocycles. In Sec. 6 the case with $N=2$ is considered. It is shown that we may construct two hidden supercharges for each of the $l$ cocycles of the Lie algebra $\mathcal{G}$.

\section{A particle model with bosonic and fermionic degrees of freedom}

Let $G$ be a compact, simple Lie group of algebra $\mathcal{G},\left[X_{i}, X_{j}\right]=i f_{i j k} X_{k}$. We set out from the superspace Lagrangian

$$
\mathcal{L}=\frac{1}{2} i \dot{\Phi}_{i} D \Phi_{i}+i q D \Phi_{i} A_{i}(\Phi)=K+\theta L, \quad i=1, \ldots, \operatorname{dim} \mathcal{G}
$$


where $\theta$ is a real Grassmann variable, the $\Phi_{i}=\Phi_{i}(t, \theta)$ are scalar superfields, and the covariant derivative $D$ and the generator of supersymmetry $Q$ are given by

$$
\Phi_{i}(t, \theta)=x_{i}(t)+i \theta \psi_{i}(t), \quad D=\partial_{\theta}-i \theta \partial_{t}, \quad Q=\partial_{\theta}+i \theta \partial_{t} .
$$

The Lagrangian is invariant under the (real, adjoint) action of $G$. Using

$$
\begin{aligned}
D \Phi_{i} & =i\left(\psi_{i}-\theta \dot{x}_{i}\right), \\
A_{i}(\Phi) & =A_{i}(x)+i \theta \psi_{j} \partial_{j} A_{i}(x),
\end{aligned}
$$

the expansion (4) of $\mathcal{L}$ gives

$$
\begin{aligned}
K & =-\frac{1}{2} \dot{x}_{i} \psi_{i}-q \psi_{i} A_{i}, \\
L & =\frac{1}{2} \dot{x}_{i} \dot{x}_{i}+\frac{1}{2} i \psi_{i} \dot{\psi}_{i}+q \dot{x}_{i} A_{i}-\frac{1}{2} i q F_{i j} \psi_{i} \psi_{j},
\end{aligned}
$$

where

$$
F_{i j}=\partial_{i} A_{j}-\partial_{j} A_{i}
$$

We easily find the momenta and canonical commutators

$$
p_{i}=\frac{\partial L}{\partial \dot{x}_{i}}=\dot{x}_{i}+q A_{i}, \quad\left[x_{i}, p_{j}\right]=i \delta_{i j}, \quad\left\{\psi_{i}, \psi_{j}\right\}=\delta_{i j},
$$

and compute

$$
H=\frac{1}{2} \dot{x}_{i} \dot{x}_{i}+\frac{1}{2} i q F_{i j} \psi_{i} \psi_{j} .
$$

The standard supersymmetry that leaves the action for (四) invariant is $\delta \Phi_{i}=$ $-i \epsilon Q \Phi_{i}$, i.e.

$$
\delta x_{i}=-i \epsilon \psi_{i}, \quad \delta \psi_{i}=\epsilon \dot{x}_{i}
$$

Noether's theorem

$$
-i \epsilon Q=\delta x_{i} p_{i}+\delta \psi_{i} \frac{\partial L}{\partial \dot{\psi}_{i}}-i \epsilon K
$$

where the piece depending on $K$ comes from the quasi-invariance of the Lagrangian, gives for the conserved supercharge

$$
Q=\dot{x}_{i} \psi_{i}
$$

It is easy to check that $Q$ above generates (12) by means of the canonical formalism, and that

$$
Q^{2}=H
$$

reproduces the right hand side of (11). 


\section{Hidden supersymmetries in the free case}

\subsection{The case of $\tilde{Q}_{3}$}

Here we shall put $q=0$ in expressions in Sec. 2. We intend first to seek an additional supersymmetry $\tilde{Q}_{3}$ such that $\left\{Q, \tilde{Q}_{3}\right\}=0$ in the form

$$
\tilde{Q}_{3}=\dot{x}_{i} f_{i j} \psi_{j}-\frac{1}{3} i f_{i j k} \psi_{i} \psi_{j} \psi_{k} \quad
$$

where $f_{i j}$ is to be determined. Since it is easier to work classically, we use the Dirac bracket formalism corresponding to (10), so that for $F$ and $G$ functions of dynamical variables

$$
\{F, G\}=\frac{\partial F}{\partial x_{l}} \frac{\partial G}{\partial p_{l}}-\frac{\partial F}{\partial p_{l}} \frac{\partial G}{\partial x_{l}}+i(-1)^{F} \frac{\partial F}{\partial \psi_{l}} \frac{\partial G}{\partial \psi_{l}} .
$$

Using $Q=p_{i} \psi_{i}$ from (14) and (16), we get

$$
\left\{Q, \tilde{Q}_{3}\right\}=-\psi_{l} \dot{x}_{i} f_{i j, l} \psi_{j}-i \dot{x}_{l}\left(\dot{x}_{i} f_{i l}-i f_{l j k} \psi_{j} \psi_{k}\right)
$$

where $f_{i j, l}=\frac{\partial}{\partial x^{l}} f_{i j}$. The second term in the r.h.s. of (18) is zero if

$$
f_{i j}=-f_{j i}
$$

and the other two cancel if

$$
f_{i[j, l]}=f_{i j l} .
$$

We could even have written $f_{i j, k}$ instead of $f_{i j k}$ in (16), and then $f_{i j, k}$ may effectively be replaced by $f_{[i j, k]}$ in virtue of the $\psi_{i} \psi_{j} \psi_{k}$ factor. Then (20) is

$$
f_{i[j, l]}=f_{[i j, l]},
$$

so that

$$
\partial_{(l} f_{i) j}=0
$$

or

$$
2 f_{i j, l}=f_{l i, j}+f_{j l, i} .
$$

Equations (21) or (22) state that the derivative of the antisymmetric tensor (19) is also skewsymmetric, and characterise $f_{i j}$ as Killing-Yano tensor [3, 4]. One way to satisfy this condition sets

$$
f_{i j}=f_{i j k} x_{k}
$$


giving

$$
\tilde{Q}_{3}=L_{i} \psi_{i}+\frac{2}{3} S_{i} \psi_{i}
$$

where

$$
L_{i}=f_{i j k} x_{j} \dot{x}_{k} \quad, \quad S_{i}=-\frac{1}{2} i f_{i j k} \psi_{j} \psi_{k} .
$$

Furthermore,

$$
\begin{aligned}
{\left[L_{i}, x_{j}\right] } & =i f_{i j k} x_{k}, \quad\left[S_{i}, \psi_{j}\right]=i f_{i j k} \psi_{k} \\
{\left[\tilde{Q}_{3}, x_{i}\right] } & =i f_{i j k} x_{j} \psi_{k}, \quad\left\{\tilde{Q}_{3}, \psi_{i}\right\}=L_{i}+2 S_{i} .
\end{aligned}
$$

Both $L_{i}$ and $S_{i}$ are representations of the Lie algebra $\mathcal{G}$ since they obey

$$
\left[L_{i}, L_{j}\right]=i f_{i j k} L_{k} \quad, \quad\left[S_{i}, S_{j}\right]=i f_{i j k} S_{k} .
$$

We note that the $\tilde{Q}_{3}$ supersymmetry does not close on the Hamiltonian, but instead we have

$$
\left\{\tilde{Q}_{3}, \tilde{Q}_{3}\right\}=\vec{J}^{2}+\frac{1}{3} \vec{S}^{2} \quad, \quad J_{i}=L_{i}+S_{i},
$$

where $J_{i}$ is the conserved charge associated with the $G$-invariance of the action (4). The result here parallels the result of [8] for particle motion in the background field of a Dirac monopole.

We note in passing also that $\tilde{Q}_{3}$ looks similar to the Kostant fermionic operator $K$ [11, 12]

$$
K=\rho_{i} \gamma^{i}-\frac{i}{3 ! 2} f_{i j k} \gamma^{i} \gamma^{j} \gamma^{k},
$$

where $\rho$ refers to some representation of $\mathcal{G}\left(c f L_{i}\right.$ in (26) ). In (30) the quantised fermion operators $\psi_{i}$ have been represented, $\psi_{i} \mapsto \frac{1}{\sqrt{2}} \gamma_{i}$, by the Dirac matrices of a euclidean space of dimension $\operatorname{dim} \mathcal{G}$ which obey $\left\{\gamma_{i}, \gamma_{j}\right\}=$ $2 \delta_{i j}$. However, (30) implies $K^{2}=\vec{\rho}^{2}+\frac{1}{3} \vec{S}^{2}$. Clearly $K$ is not proportional the supercharge $\tilde{Q}_{3}$ of (16), for any representation $\rho$ of $\mathcal{G}$.

\subsection{Other hidden supersymmetries}

We generalise now the previous paragraph to supercharges involving the higher order cocycles of the Lie algebra $\mathcal{G}$. Instead of (16), we consider $\tilde{Q}_{5}$ as in (3)

$$
\tilde{Q}_{5}=p_{i} f_{i j k p} \psi_{j k p}-\frac{1}{5} i \Omega_{i j k p q} \psi_{i j k p q},
$$


where $f_{i j k p}=f_{i j k p}(x)$,

$$
f_{i j k p}=f_{i[j k p]},
$$

we have used the abbreviation $\psi_{i j k \ldots}=\psi_{i} \psi_{j} \psi_{k} \ldots$, and $\Omega_{i j k p q}$ is by definition totally antisymmetric in all its five indices. Instead of (32) we might consider the replacement of $\Omega_{i j k p q}$ by

$$
f_{[i j k p, q]},
$$

where $f_{i j k p, q}=\partial f_{i j k p} / \partial x_{q}$. We now demand that $\tilde{Q}_{5}$ anticommutes with $Q=p_{i} \psi_{i}$

$$
\left\{Q, \tilde{Q}_{5}\right\}=0
$$

quantum mechanically for variety, and also because promotion of the classical calculation in this context is not in this instance trivial. Thus

$$
\begin{aligned}
\left\{Q, \tilde{Q}_{5}\right\} & =p_{l}\left\{\psi_{l}, \tilde{Q}_{5}\right\}+\left[\tilde{Q}_{5}, p_{l}\right] \psi_{l} \\
& =p_{l}\left(3 p_{i} f_{i j k l} \psi_{j k}-i \Omega_{l j k p q} \psi_{j k p q}\right)+i p_{l} f_{l j k p, q} \psi_{j k p q}
\end{aligned}
$$

The first term of (35) can be eliminated by requiring that $f_{i j k l}$ is antisymmetric in $i$ and $l$, and, hence, using (33) that

$$
f_{i j k p}=f_{[i j k p]} .
$$

The other result needed to secure (34) is the vanishing of the part of

$$
f_{l j k p, q}-\Omega_{l j k p q}
$$

antisymmetric in $j k p q$. If we try the ansatz

$$
f_{l j k p}=\Omega_{l j k p q} x_{q}
$$

then this requirement is satisfied. Then

$$
\tilde{Q}_{5}=p_{i} \Omega_{i j k p q} x_{q} \psi_{j k p}-\frac{1}{5} i \Omega_{i j k p q} \Psi_{i j k p q}
$$

is a hidden, $A d G$-invariant supercharge. This situation is one that applies to $\tilde{Q}_{7}$, involving $\Omega_{7}$, etc. Actually, since the charges are constructed using the structure constants and the higher-order cocycles, there will be a hidden supercharge for each cocycle in a model with an arbitrary simple compact group. We may then conclude the following:

The $A d(G)$-invariant free supersymmetric particle model described by $\mathcal{L}=$ $\frac{1}{2} \Phi_{i} D \Phi_{i}$ admits $l$ hidden supercharges $\tilde{Q}_{s}, s=1, \ldots, l$. These are determined 
by the l Lie algebra cohomology cocycles of the simple compact algebra $\mathcal{G}$ of rank $l$.

If we had written $f_{[i j k p, q]}$ in the second term of (31), then (37) corresponds to the vanishing of the part of

$$
f_{l[j k p], q}=f_{[l j k p, q]}
$$

antisymmetric in $j k p q$. To extract the minimal condition, we write

$$
f_{[l j k p, q]}=\frac{4}{5} f_{l[j k p, q]}+\frac{1}{5} f_{[j k p q], l} .
$$

Then (40) is equivalent to

$$
f_{[l j k p, q]}=f_{[j k p q], l \quad},
$$

which is an analogue of (23) and, together with the complete antisymmetry of $f_{i j k l}$, means that $f_{i j k l}$ is a Killing-Yano tensor of valence four. This is consistent with Tanimoto's analysis [6], although in a slightly different context. The previous result may be rephrased in the following form:

The additional supersymmetry exists because each Lie algebra cocycle of order $2 m-1$ provides a Killing-Yano tensor of valence $2(m-1)$ by the analogous to (38) since then

$$
f_{i i_{1} \ldots i_{2 m-3}, q}+f_{q i_{1} \ldots i_{2 m-3}, i}=0 \quad .
$$

One might look for different solutions, but we did not find any. For example, the condition (42) defeats the otherwise interesting ansatz $f_{i j k l}=$ $f_{[i j} f_{k l]}$, where $f_{i j}$ is a second order Killing-Yano tensor. In fact, the KillingYano condition appears to be very restrictive, and it is likely that (24) and (38) are the only possible solutions for the cases considered.

\section{$4 \tilde{Q}_{3}$ in the presence of a background field}

We investigate now to what extent it is possible to reproduce the analysis of sec. 3 in the $A \neq 0$ case. When $Q$ is given by (14) and $\tilde{Q}_{3}$ by (16), where now $p_{i}=\dot{x}_{i}+q A_{i}$ as in Sec. 2, we find classically that $\left\{Q, \tilde{Q}_{3}\right\}=0$ requires

$$
\begin{aligned}
\left\{Q, \tilde{Q}_{3}\right\}= & -\psi_{l} \dot{x}_{i} f_{i j, l} \psi_{j}-i \dot{x}_{l}\left(\dot{x}_{i} f_{i l}-i f_{l j k} \psi_{j} \psi_{k}\right) \\
& +q f_{l j} F_{i l} \psi_{i} \psi_{j}=0
\end{aligned}
$$


so we have to impose (19), (23) and

$$
f_{l[j} F_{i] l}=0
$$

where $F_{i j}$ is given by $(\mathrm{g})$. It is sensible to try first the solution

$$
f_{i j}=f_{i j k} x_{k}
$$

of (19) and (23). To satisfy (45) we may choose

$$
F_{i j}=x_{i} y_{j}-x_{j} y_{i}
$$

where $y_{i}=d_{i j k} x_{j} x_{k}$, whenever there exists an invariant symmetric tensor $d_{i j k}$ on the algebra (which excludes $s u(2))$. This is true because of the identities $f_{i j k} x_{j} x_{k}=0=f_{i j k} x_{j} y_{k}$, the last one due to the invariance of the symmetric tensor $d_{i j k}$. However consistency with (9) demands that any ansatz for $F_{i j}$, and (47) in particular, obeys

$$
\partial_{[i} F_{j k]}=0
$$

The simple ansatz $F_{i j}=a(Z) f_{i j k} x_{k}$ where $Z=x_{k} x_{k}$, satisfies (48) when the algebra is $s u(2)$, in which case $a(Z) \propto Z^{-3 / 2}$, as is expected for the monopole [8]. But for $s u(n)$, e.g. for $s u(3)$, for which $Z=x_{k} x_{k}$ and $Y=$ $d_{i j k} x_{i} x_{j} x_{k}=x_{k} y_{k}$, the ansatz $F_{i j}=a(Z, Y) f_{i j k} x_{k}$ fails. We can see this by contracting

$$
0=\partial_{[i} F_{j k]}=2 \frac{\partial a}{\partial Z} x_{[i} f_{j k] l} x_{l}+3 \frac{\partial a}{\partial Y} y_{[i} f_{j k] l} x_{l}+a f_{i j k},
$$

with $y_{i}$, which gives

$$
\frac{2}{3} Y \frac{\partial a}{\partial Z} f_{j k l} x_{l}+y^{2} \frac{\partial a}{\partial Y} f_{j k l} x_{l}+a f_{j k l} y_{l}=0 .
$$

The independence of the tensors $f_{i j k} x_{l}$ and $f_{j k l} y_{l}$ now gives $a=0$. The method used here can be extended to show the failure also of the more general ansatz

$$
F_{i j}=a(Z, Y) f_{i j k} x_{k}+b(Z, Y) f_{i j k} y_{k} .
$$

The case of $s u(2)$ of course escapes such a failure because $y_{i}$ then does not exist. 
The choice (47) can be used for $s u(n), n \geq 3$ : it obeys (48) directly but also allows us to write down suitable choices of $A_{i}$ for which (9) reproduces (47). For example, we may use

$$
A_{i}=-\frac{1}{3} Y x_{i}
$$

More generally, any $A_{i}$ of the form

$$
A_{i}=\alpha(Z, Y) x_{i}+\beta(Z, Y) y_{i}
$$

gives $F_{i j}$ in suitable form:

$$
F_{i j}=\left(2 \frac{\partial \beta}{\partial Z}-3 \frac{\partial \alpha}{\partial Y}\right)\left(x_{i} y_{j}-x_{j} y_{i}\right)
$$

Thus, for any $G$-invariant model (4) there exists an additional supersymmetry $\tilde{Q}_{3}$ given by

$$
\tilde{Q}_{3}=p_{i} f_{i j k} \psi_{j} x_{k}-\frac{1}{3} i f_{i j k} \psi_{i} \psi_{j} \psi_{k},
$$

determined by the structure constants $f_{i j k}$ of $\mathcal{G}$. The contribution proportional to $A_{i}$ disappears from the first term of (54) again because of the identities $f_{i j k} x_{j} x_{k}=0=f_{i j k} x_{j} y_{k}$. But this does not mean that we have recovered the free case, because $A_{i}$ is present in $Q=\left(p_{i}-q A_{i}\right) \psi_{i}$. $\tilde{Q}$ satisfies the relations

$$
\left[\tilde{Q}_{3}, x_{i}\right]=i f_{i j k} x_{j} \psi_{k},\left\{\tilde{Q}_{3}, \psi_{i}\right\}=L_{i}+2 S_{i},\left\{\tilde{Q}_{3}, \tilde{Q}_{3}\right\}=(\vec{L}+\vec{S})^{2}+\frac{1}{3} \vec{S}^{2}
$$

where now $L_{i}=f_{i j k} x_{j} p_{k}$.

We have not found, however, an analogue of $\tilde{Q}_{5}$ for $q \neq 0$. If the KillingYano tensor is $f_{i j k l}=\Omega_{i j k l m} x_{m}$ and $F_{i j}$ is proportional to (47), the condition corresponding to (45),

$$
f_{l[j m n} F_{i] l}=0,
$$

is not satisfied because, in contrast with $f_{i j k} x_{j} y_{k}=0, \Omega_{i j k l m} x_{l} y_{m} \neq 0$. It is possible that the use of more general background fiels (see [13]) opens the way to richer possibilities.

\section{$5 \quad N=1$ Fermion superfields}




\subsection{Basic formalism}

We turn now to a different supersymmetric model in which hidden supersymmetries related to higher order cocycles also occur. This model is a theory of fermions with all states in one energy level, and without bosonic dynamical variables [0]. Consider the $A d(S U(n))$-invariant superspace Lagrangian given by

$$
\mathcal{L}=\frac{1}{2} \Lambda_{i} D \Lambda_{i}+\frac{1}{3 !} i g f_{i j k} \Lambda_{i} \Lambda_{j} \Lambda_{k}
$$

where the $\Lambda_{i}=\Lambda_{i}(t, \theta)$ are $i=1, \ldots, \operatorname{dim} \mathcal{G}$ fermionic superfields,

$$
\Lambda_{i}(t, \theta)=\psi_{i}(t)+\theta B_{i}(t), \quad D \Lambda_{i}=B_{i}-i \theta \dot{\psi}_{i} .
$$

The expansion of (58) may be written as $\mathcal{L}=K+\theta L$, where now

$$
\begin{aligned}
K & =\frac{1}{2} \psi_{i} B_{i}+\frac{1}{3 !} i g \psi_{i} \psi_{j} \psi_{k}, \\
L & =\frac{1}{2}\left(i \dot{\psi}_{i} \psi_{i}+B_{i} B_{i}+i g f_{i j k} \psi_{i} \psi_{j} B_{k}\right) .
\end{aligned}
$$

As in Sec. 2, we may use the expression of $K$ and $L$ in Noether's theorem (see (13)) for the variations

$$
\delta \psi_{i}=-\epsilon B_{i}, \quad \delta B_{i}=i \epsilon \dot{\psi}_{i}
$$

to obtain

$$
Q=\frac{1}{6} i g f_{i j k} \psi_{i} \psi_{j} \psi_{k}
$$

All the bosonic components $B_{i}$ are auxiliary. Using their Euler-Lagrange equations to solve for $B_{i}$, one obtains the classical Lagrangian $L_{c}=\frac{1}{2} i \psi_{i} \dot{\psi}_{i}$. The classical Hamiltonian vanishes identically \&, but the quantum Hamiltonian, defined by $H=Q^{2}$, and computed using $\left\{\psi_{i}, \psi_{j}\right\}=\delta_{i j}$, is not zero but a constant,

$$
Q^{2}=\frac{1}{48} g^{2} n\left(n^{2}-1\right)
$$

for $s u(n)$.

\subsection{Hidden supersymmetries in the fermionic model}

As for the (4) model for $q=0$, there exist in this case additional supercharges $\tilde{Q}$ for every non-trivial cocycle of any $s u(n)$ Lie algebra. To see this, let

\footnotetext{
${ }^{2}$ There is an $S_{i} S_{i}$ part, which is proportional to $f_{m i j} f_{m k l} \psi_{i} \psi_{j} \psi_{k} \psi_{l}$ and which is zero classically by virtue of the Jacobi identity and the fermionic character of the $\psi$ 's.
} 
$\Omega_{i_{1} \ldots i_{2 m-1}}^{(2 m-1)}$ be a non-trivial cocycle corresponding to an invariant symmetric tensor $t$ of order $m$. If $t$ has components $t_{l_{1} \ldots l_{m}}$, then the $(2 m-1)$-order cocycle is given by

$$
\Omega_{i_{1} \ldots i_{2 m-1}}^{(2 m-1)}=f_{\left[i_{1} i_{2}\right.}^{l_{i}} \ldots f_{i_{2 m-3} i_{2 m-2}}^{l_{m-1}} t_{\left.i_{2 m-1}\right] l_{i} \ldots l_{m-1}} .
$$

Using it, we form

$$
\tilde{Q}_{2 m-1}=\Omega_{i_{1} \ldots i_{2 m-1}}^{(2 m-1)} \psi_{i_{1} \ldots i_{2 m-1}}
$$

where

$$
\psi_{i_{1} \ldots i_{2 m-1}} \equiv \psi_{i_{1}} \ldots \psi_{i_{2 m-1}} .
$$

We compute the quantum anticommutator of $Q$ in (62) and $\tilde{Q}_{2 m-1}$ by expressing the products $\psi_{i_{i} i_{2} i_{3}} \psi_{j_{1} \ldots j_{2 m-1}}$ and $\psi_{j_{1} \ldots j_{2 m-1}} \psi_{i_{i} i_{2} i_{3}}$ as linear combinations of completely antisymmetrised products of $\psi$ 's. This can be done by repeated use of the identities (deduced from $\left\{\psi_{i}, \psi_{j}\right\}=\delta_{i j}$ )

$$
\begin{aligned}
\psi_{i} \psi_{j_{1} \ldots j_{p}} & =\psi_{i j_{1} \ldots j_{p}}+\frac{1}{2} p \delta_{\left[i j_{1}\right.} \psi_{\left.j_{2} \ldots j_{p}\right]}, \\
\psi_{j_{1} \ldots j_{p}} \psi_{i} & =\psi_{j_{1} \ldots j_{p} i}+\frac{1}{2} p \psi_{\left[j_{1} \ldots j_{p-1}\right.} \delta_{\left.j_{p}\right] i} .
\end{aligned}
$$

Then we easily find that the terms with no $\delta$ 's or an even number of them vanish identically because the two contributions coming from the anticommutator cancel each other. So we are left with

$$
\begin{aligned}
\left\{Q, \tilde{Q}_{2 m-1}\right\}= & \frac{1}{6} i g\left[3(2 m-1) f_{k i_{2} i_{3}} \Omega_{k j_{2} \ldots j_{2 m-1}}^{(2 m-1)} \psi_{i_{2} i_{3} j_{2} \ldots j_{2 m-1}}\right. \\
& \left.-\frac{1}{4} \frac{(2 m-1) !}{(2 m-4) !} f_{k l m} \Omega_{k l m j_{4} \ldots j_{2 m-1}}^{(2 m-1)} \psi_{j_{4} \ldots j_{2 m-1}}\right] .
\end{aligned}
$$

The first term in (68) vanishes due to the Jacobi identity (since the indices $i_{2} i_{3} j_{2} \ldots j_{2 m-1}$ are antisymmetrised due to the presence of the $\psi$ 's ) and the second also vanishes because the maximal contraction of indices among the above two $s u(n)$ cocycles of different order gives zero [10. Hence, the $l \tilde{Q}_{2 m-1}$ define new conserved fermionic charges of higher order. As in the case of $Q$ in (62), they square to a constant. For example, let us consider the case of $\tilde{Q}_{5}$ for $s u(n), n \geq 3$. The square of $\tilde{Q}_{5}$ is given by

$$
\tilde{Q}_{5}^{2}=\Omega_{i_{1} \ldots i_{5}}^{5} \Omega_{j_{1} \ldots j_{5}}^{5} \psi_{i_{1} \ldots i_{5}} \psi_{j_{1} \ldots j_{5}} .
$$

It is shown in [16] (see also [10]) that this square is a number. Hence, $Q, \tilde{Q}_{5}$ and I close into a superalgebra.

\footnotetext{
${ }^{3}$ For the relation among symmetric tensors and cocycles of generic compact simple Lie algebra $\mathcal{G}$ see [14], 10] and [15].
} 


\section{$6 \quad N=2$ Fermion superfields}

\subsection{Basic formalism}

We now consider a purely fermionic model with two standard supersymmetries [7] (see also [17]). The supersymmetry algebra in terms of the covariant derivatives for this model is

$$
D=\partial_{\theta}-i \theta^{*} \partial_{t}, \quad D^{*}=-\partial_{\theta^{*}}+i \theta \partial_{t}, \quad\left\{D, D^{*}\right\}=2 i \partial_{t} .
$$

The $N=1$ superfields $\Lambda_{i}$ are replaced by $N=2$ superfields $\Psi_{i}=\Psi_{i}\left(t, \theta, \theta^{*}\right)$, $i=1, \ldots, \operatorname{dim} \mathcal{G}$, to which the chirality condition $D^{*} \Psi_{i}=0$ is imposed. This of course means that $\Psi_{i}^{*}$ obeys $D \Psi_{i}^{*}=0$ and is antichiral. Solving as usual the chirality condition we obtain the superfield expansions

$$
\begin{aligned}
\Psi_{i} & =e^{i \theta^{*} \theta \partial_{t}}\left(\mu_{i}-\theta B_{i}\right)=\mu_{i}-\theta B_{i}+i \theta^{*} \theta \dot{\mu}_{i} \\
\Psi_{i}^{*} & =\mu_{i}^{*}-\theta^{*} B_{i}^{*}-i \theta^{*} \theta \dot{\mu}_{i}^{*},
\end{aligned}
$$

where $\mu_{i}$ are fermionic and $B_{i}$ are bosonic. The following $A d(G)$-invariant superspace action has the property that the $B$ 's are non-dynamical, and includes an interaction term:

$$
\begin{aligned}
S= & -\frac{1}{2} \int d t d \theta d \theta^{*} \Psi_{i} \Psi_{i}^{*} \\
& +\frac{1}{6} \int d t d \theta i C_{i j k} \Psi_{i} \Psi_{j} \Psi_{k}+\frac{1}{6} \int d t d \theta^{*} i C_{i j k} \Psi_{i}^{*} \Psi_{j}^{*} \Psi_{k}^{*},
\end{aligned}
$$

where $C_{i j k}$ are the structure constants of the Lie algebra $\mathcal{G}$. The $\operatorname{Ad}(G)$ invariant component Lagrangian is given by

$$
L=\frac{1}{2} i\left(\mu_{i}^{*} \dot{\mu}_{i}+\mu_{i} \dot{\mu}_{i}^{*}\right)+\frac{1}{2} B_{i}^{*} B_{i}-\frac{1}{2} i C_{i j k} \mu_{i} \mu_{j} B_{k}-\frac{1}{2} i C_{i j k} \mu_{i}^{*} \mu_{j}^{*} B_{k}^{*} .
$$

The Euler Lagrange equations of the $B_{i}, B_{i}^{*}$ are algebraic and can be used to eliminate these variables. Then, by writing $J_{i}=-\frac{1}{2} i C_{i j k} \mu_{j} \mu_{k}$, the Lagrangian becomes

$$
L=\frac{1}{2} i\left(\mu_{i}^{*} \dot{\mu}_{i}+\mu_{i} \dot{\mu}_{i}^{*}\right)-2 J_{i} J_{i}^{*} .
$$

The canonical formalism yields the Dirac brackets

$$
\left\{\mu_{i}, \mu_{j}^{*}\right\}=-i \delta_{i j}, \quad\left\{\mu_{i}, \mu_{j}\right\}=0, \quad\left\{\mu_{i}^{*}, \mu_{j}^{*}\right\}=0,
$$

and classically we have

$$
H=2 J_{i} J_{i}^{*}
$$


The non-zero supersymmetry variations of the fields $\mu_{i}$ and $B_{i}$ are given by

$$
\begin{aligned}
\delta_{\epsilon^{*}} B_{i} & =-2 i \epsilon^{*} \dot{\mu}_{i}, \quad \delta_{\epsilon^{*}} \mu_{i}^{*}=\epsilon^{*} B_{i}^{*}, \\
\delta_{\epsilon} B_{i}^{*} & =-2 i \epsilon \dot{\mu}_{i}^{*}, \quad \delta_{\epsilon} \mu_{i}=\epsilon B_{i} .
\end{aligned}
$$

These variations correspond, via Noether's theorem, to the conserved charges

$$
Q=\frac{1}{3} i C_{i j k} \mu_{i} \mu_{j} \mu_{k}, \quad Q^{*}=\frac{1}{3} i C_{i j k} \mu_{i}^{*} \mu_{j}^{*} \mu_{k}^{*}
$$

When we quantise the theory we shall regard the $\mu_{i}$ as the creation operators. Hence, to avoid confusion, the following replacements will be made from now on: $\mu_{i}^{*}=\pi_{i}, \mu_{i}=c_{i}$, as in [17], so that $\pi^{*}=c_{i}$. Thus in the quantum theory, we have the anticommutation relations

$$
\left\{c_{i}, \pi_{j}\right\}=\delta_{i j}
$$

Also the quantum mechanical Hamiltonian is defined via

$$
\left\{Q, Q^{*}\right\}=2 H_{q}
$$

which gives the result

$$
H_{q}=\left\{J_{i}, J_{i}^{*}\right\}-\frac{1}{6} c^{2},
$$

where $c^{2}=C_{i j k} C_{i j k}\left(=n\left(n^{2}-1\right)\right.$ for $\left.s u(n)\right)$, and where it should be noted that $J_{i}$ and $J_{i}^{*}$ do not commute. One might expect that that $H_{q}$ is closely related to the quadratic Casimir operator $X^{2}=X_{i} X_{i}$ of $\mathcal{G}$, where $X_{i}=-i C_{i j k} c_{j} \pi_{k}$. It is simple to confirm this for the case of $s u(n)$ by proving the following identities

$$
\begin{aligned}
X_{i} X_{i} & =n N-2 J_{i} J_{i}^{*} \\
& =n\left(n^{2}-1-N\right)-2 J_{i}^{*} J_{i},
\end{aligned}
$$

where $N=c_{i} \pi_{i}$ is the total fermion number operator, and $\pi_{i} c_{i}=\left(n^{2}-1-\right.$ $N)$. These allow the commutator and anticommutator of $J_{i}$ and $J_{i}^{*}$ to be calculated, and give rise to the result

$$
H_{q}=\frac{1}{3} n\left(n^{2}-1\right)-X_{i} X_{i} .
$$

The results (81) and (83), viewed together, seem a strangely related pair. However their agreement, as well as the correctness of (82), can easily be confirmed by considering actions of the operators in question on each of the 
fermion number $N=0,1,2,3$ states of the simple but non-trivial $S U(2)$ version of the theory. Further, having set out from the definition (80) of $H_{q}$, we know that all energy eigenvalues are non-negative.

In addition to

$$
q_{30}=Q / 2=\frac{1}{3 !} i C_{i j k} c_{i} c_{j} c_{k} \quad, \quad q_{03}=q_{30}^{*}=Q^{*} / 2,
$$

in which the first and second subscripts indicate the numbers of $c_{i}$ and $\pi_{i}$ factors respectively, two further fermionic operators occur naturally:

$$
q_{21}=\frac{1}{2} i C_{i j k} c_{i} c_{j} \pi_{k}, \quad q_{12}=\frac{1}{2} i C_{i j k} c_{i} \pi_{j} \pi_{k} .
$$

These operators each anticommute with each of $q_{30}$ and $q_{03}$ and obey

$$
\left\{q_{21}, q_{12}\right\}=\frac{1}{2} X_{i} X_{i} \quad, \quad q_{21}^{2}=0 \quad, \quad q_{12}^{2}=0 .
$$

It follows that $q_{21}$ and $q_{12}$ commute with $X_{i} X_{i}$ and with $H$.

We have found a second supersymmetry which anticommutes with the original one; its closure does not give a new operator independent of $H$. However, in view of the results (81) and (83), it is not appropriate to say that our theory has $N=4$ supersymmetry. We have simply found two additional supercharges naturally associated with the structure constants of $\mathcal{G}$, a consideration that is built on significantly in the next subsection.

\subsection{Hidden supersymmetries}

One obvious question asks whether it is possible to find new supercharges that generalize those of Sec. 6.1. Consider the case of charges constructed using the five-cocycle $\Omega_{i_{1} \ldots i_{5}}$ rather than the three-cocycle $C_{i j k}$. An analysis of the possibilities, $q_{50}, q_{41}, q_{32}, q_{23}, q_{14}, q_{05}$, leads one to conclude that only

$$
q_{05}=\frac{1}{5 !} i \Omega_{i_{1} \ldots i_{5}} \pi_{i_{1}} \ldots \pi_{i_{5}}, \quad q_{50}=\frac{1}{5 !} i \Omega_{i_{1} \ldots i_{5}} c_{i_{1}} \ldots c_{i_{5}}
$$

are hidden conserved supercharges because only they anticommute with $q_{21}$ and $q_{12}$.

Moreover, we have the following general result:

The $l=\operatorname{rank} \mathcal{G}$ pairs of fermionic charges

$$
\begin{aligned}
q_{0,2 m-1} & =\frac{i}{(2 m-1) !} \Omega_{i_{1} \ldots i_{2 m-1}} \pi_{i_{1}} \ldots \pi_{i_{2 m-1}} \\
q_{2 m-1,0} & =\frac{i}{(2 m-1) !} \Omega_{i_{1} \ldots i_{2 m-1}} c_{i_{1}} \ldots c_{i_{2 m-1}}
\end{aligned}
$$


determined by the $(2 m-1)$-cocycles of the Lie algebra $\mathcal{G}$, where the allowed values of $m$ depend on the specific $\mathcal{G}$ considered, also commute with $q_{21}$ and $q_{12}$, and hence they commute with $H_{q}$ and are conserved supercharges.

Proof: Let us restrict ourselves to $q_{2 m-1,0}$ (the case $q_{0,2 m-1}$ is completely analogous). Consider first

$$
\left\{q_{2 m-1,0}, q_{12}\right\} \propto \Omega_{i\left[i_{2} \ldots i_{2 m-1}\right.} C_{\left.j_{1} j_{2}\right] i} c_{j_{1}} c_{j_{2}} c_{i_{2}} \ldots c_{i_{2 m-1}}
$$

where the antisymmetrization is forced by the presence of the $c$ 's. This expression vanishes by the $G$-invariance of $\Omega$, since this implies

$$
\Omega_{i\left[i_{1} \ldots i_{2 m-2}\right.} C_{\left.i_{2 m-1}\right] i j}=0 .
$$

Hence, $\left\{q_{2 m-1,0}, q_{12}\right\}=0$. Now we have to check that the following anticommutator also vanishes:

$$
\begin{aligned}
\left\{q_{2 m-1,0}, q_{21}\right\} \propto & \Omega_{i i_{2} \ldots i_{2 m-1}} C_{i j_{1} j_{2}} c_{j_{1}} c_{i_{2}} \ldots c_{i_{2 m-1}} \pi_{j_{2}} \\
& +(m-1) \Omega_{i j i_{3} \ldots i_{2 m-1}} C_{i j j_{1}} c_{j_{1}} c_{i_{3}} \ldots c_{i_{2 m-1}}
\end{aligned}
$$

The first term vanishes due to the antisymmetry in the indices $j_{1}, i_{2}, \ldots, i_{2 m-1}$ and the invariance of $\Omega$. To show that the second term also vanishes, we have to prove that

$$
D \equiv \Omega_{i j i_{1} \ldots i_{2 m-3}} C_{i_{2 m-2} i j} c_{i_{1}} \ldots c_{i_{2 m-2}}
$$

is equal to zero. Indeed, the invariance of $\Omega$ allows us to write it in terms of the $C$ 's and the invariant symmetric tensor $t_{l_{1} \ldots l_{m}}$ (see (64)) without having to involve $i, j$ in the antisymmetrization, so we have

$$
D=C_{i i_{1} l_{1}} C_{i_{2} i_{3} l_{2}} \ldots C_{i_{2 m-4} i_{2 m-3} l_{m-1}} t_{l_{1} \ldots l_{m-1} j} C_{i_{2 m-2} i j} c_{i_{1}} \ldots c_{i_{2 m-2}} .
$$

Now, using the Jacobi identity

$$
C_{i i_{1} l_{1}} C_{i_{2 m-2} i j}=C_{i i_{2 m-2} l_{1}} C_{i_{1} i j}+C_{i i_{1} i_{2 m-2}} C_{l_{1} i j}
$$

we arrive at

$$
\begin{aligned}
D= & C_{i i_{2 m-2} l_{1}} C_{i_{2} i_{3} l_{2}} \ldots C_{i_{2 m-4} i_{2 m-3} l_{m-1}} t_{l_{1} \ldots l_{m-1} j} C_{i_{1} i j} c_{i_{1}} \ldots c_{i_{2 m-2}} \\
& +C_{i i_{1} i_{2 m-2}} C_{i_{2} i_{3} l_{2}} \ldots C_{i_{2 m-4} i_{2 m-3} l_{m-1}} t_{l_{1} \ldots l_{m-1} j} C_{l_{1} i j} c_{i_{1}} \ldots c_{i_{2 m-2}}
\end{aligned}
$$

The first term of this expression is equal to $-D$ due to the presence of $c_{i_{1}}$ and $c_{i_{2 m-2}}$, and the second term vanishes because $C_{l_{1} i j}$ is antisymmetric in 
$l_{1}, j$ whereas $t_{l_{1} \ldots l_{m}-1 j}$ is symmetric in these indices. So we have $D=-D$, $D=0,\left\{q_{2 m-1,0}, q_{21}\right\}=0$ and $\left[q_{2 m-1,0}, H_{q}\right]=0$, q.e.d.

Hence, the following result follows:

For every simple Lie algebra $\mathcal{G}$ of rank l, the model (79) has a series of $2 l$ conserved supercharges that are constructed from the primitive cocycles of $\mathcal{G}$, which include the supersymmetry generators.

\section{$7 \quad$ Hidden supersymmetries as Noether charges}

All the hidden supercharges discussed can be shown to be Noether charges associated with actual supersymmetries of the actions of the models in question. One way to realise this is to use the quantum commutator of the supercharge and the variables of the model to extract the variations. Explicitly, if $\tilde{Q}$ is the conserved supercharge and $u$ is a generic component field in the model, its variation may be defined by

$$
\delta_{\tilde{\epsilon}} u=[\tilde{\epsilon} \tilde{Q}, u],
$$

where $\tilde{\epsilon}$ is the corresponding fermionic parameter.

If $\tilde{Q}$ is a symmetry of the classical action $S=\int d t L, \delta_{\tilde{\epsilon}} S=0$ for the constant parameter $\tilde{\epsilon}$. This means that, if we allow $\tilde{\epsilon}$ to become a function of $t$, its variation will be of the form

$$
\delta_{\tilde{\epsilon}} S=\int d t(-i \dot{\tilde{\epsilon}} \tilde{Q})
$$

ignoring boundary terms, where $\tilde{Q}$ is the conserved Noether charge for the symmetry (96). Indeed, from (97) we get $\delta_{\tilde{\epsilon}} S=\int d t(i \tilde{\epsilon} \dot{\tilde{Q}})$, and since for solutions of the Lagrange equations $\delta_{\tilde{\epsilon}} S$ must be zero for any $\delta$, it follows that $\dot{\tilde{Q}}=0$ and $\tilde{Q}$ is the conserved charge. This procedure is particularly suitable when, as here, the complications addressed in [18] do not arise.

We now give the variations obtained by using (96). In the bosonic case with $A_{i} \neq 0$, use of (10) yields

$$
\delta_{\tilde{\epsilon}} x_{i}=-i \tilde{\epsilon}_{i} f_{i j k} x_{k} \psi_{j}, \quad \delta_{\tilde{\epsilon}} \psi_{i}=\tilde{\epsilon} \dot{x}_{j} f_{j i k} x_{k}-i \tilde{\epsilon} f_{j k i} \psi_{j} \psi_{k}
$$

for the variation induced by $\tilde{Q}_{3}$ (eq. (25)). If now we put $\epsilon=\epsilon(t)$ and ignore boundary terms in the integrand, we do find $\delta L=-i \epsilon \tilde{Q}_{3}$, recovering $\tilde{Q}_{3}$ as the Noether charge. The same applies to the other supercharges below. 
Consider $\tilde{Q}_{5}$ (eq. (31)). The corresponding formulae for the variations for the $A=0$ model of Sec. 3.2 are,

$$
\delta_{\tilde{\epsilon}} x_{i}=-i \tilde{\epsilon} \Omega_{i j k p q} x_{q} \psi_{j k p}, \quad \delta_{\tilde{\epsilon}} \psi=3 \tilde{\epsilon} \dot{x}_{j} \Omega_{j k p i q} x_{q} \psi_{k p}-i \tilde{\epsilon} \Omega_{j k p q i} \psi_{j k p q} .
$$

Note that in the above variations $\delta \psi_{i}$ involves the derivative of $\dot{x}$ (mathematically, this means that succesive tangent spaces - jet spaces - are needed to define the action of the $\tilde{Q}$ 's). This is not the case for the two purely fermionic models for which the canonical quantum commutators give

$$
\delta_{\tilde{\epsilon}} \psi_{i}=(2 m-1) \tilde{\epsilon} \Omega_{i_{1} \ldots i_{2 m-2} i} \psi_{i_{1} \ldots i_{2 m-2}}
$$

for $\tilde{Q}_{2 m-1}$ (eq. (65)), for the $N=1$ model of Sec. 5.1, and

$$
\begin{aligned}
\delta_{\tilde{\epsilon}^{*}} \mu_{i} & =\frac{i \tilde{\epsilon}^{*}}{(2 m-2) !} \Omega_{i_{1} \ldots i_{2 m-2} i} \mu_{i_{1}}^{*} \ldots \mu_{i_{2 m-2}}^{*}, \quad \delta_{\tilde{\epsilon}^{*}} \mu_{i}^{*}=0, \\
\delta_{\tilde{\epsilon}} \mu_{i}^{*} & =\frac{i \tilde{\epsilon}}{(2 m-2) !} \Omega_{i_{1} \ldots i_{2 m-2} i} \mu_{i_{1}} \ldots \mu_{i_{2 m-2}}, \quad \delta_{\tilde{\epsilon}} \mu_{i}=0
\end{aligned}
$$

for the variations produced, respectively, by the supercharges $q_{0,2 m-1}$ and $q_{2 m-1,0}$ (eqs. (88)), of the $\mathrm{N}=2$ model of Sec. 6.1.

To proceed further, consider first the closure of $\delta_{\epsilon}, \delta_{\tilde{\epsilon}}$ on $x_{i}$, say, for the $A \neq 0$ model in Sec. 4. First we find

$$
\left[\delta_{\epsilon}, \delta_{\tilde{\epsilon}}\right] x_{i}=0
$$

reflecting the fact that $\left\{Q, \tilde{Q}_{3}\right\}=0$ (eq. (44)). For $\left[\delta_{\tilde{\epsilon}^{\prime}}, \delta_{\tilde{\epsilon}}\right]$ we find

$$
\left[\delta_{\tilde{\epsilon}^{\prime}}, \delta_{\tilde{\epsilon}}\right] x_{i}=-2 i \tilde{\epsilon} \tilde{\epsilon}^{\prime} f_{i j k} J_{j} x_{k}
$$

where

$$
J_{i}=f_{i j k} x_{j} \dot{x}_{k}-\frac{1}{2} i f_{i j k} \psi_{j} \psi_{k}
$$

is the conserved charge ( $c f .(29)$ ) associated with the adjoint transformations $\delta x_{i}=f_{i j k} a_{j} x_{k}, \delta \psi_{i}=f_{i j k} a_{j} \psi_{k}$ which leave the Lagrangian (8) invariant. This of course agrees with the variations on $x_{i}$ induced by the operator $\left\{\tilde{Q}_{3}, \tilde{Q}_{3}\right\}$ expressed in the form (cf. again (29) ) $\vec{J}^{2}+\frac{1}{3} \vec{S}^{2}$. A similar analysis can be performed for the corresponding actions upon $\psi_{i}$, for which we get

$$
\left[\delta_{\tilde{\epsilon}^{\prime}}, \delta_{\tilde{\epsilon}}\right] \psi_{i}=-2 i \tilde{\epsilon} \tilde{\epsilon}^{\prime} f_{i j k} J_{j} \psi_{k}-2 i \tilde{\epsilon} \tilde{\epsilon}^{\prime} f_{j l m} f_{j i k} x_{m} x_{k} \dot{\psi}_{l}
$$

in which we see the second term is zero on shell, using $\dot{\psi}_{l}=q F_{l j} \psi_{j}$ where $F_{l j}$ is given by (47). 


\section{Concluding remarks}

We have shown in this paper that there exist 'hidden' supersymmetric fermionic charges associated with the Lie algebra cohomology cocycles of the symmetry group for three simple $A d(G)$-invariant supersymmetric models, one with bosonic and fermionic coordinates and two (for $N=1$ and $N=2$ ) with only fermionic dynamical coordinates. For the first one, and in the free case, there are $l$ additional supersymmetries the existence of which is tied to the Killing-Yano tensors of valence $(2 m-2)$ that may be constructed from the $(2 m-1)$ Lie algebra cohomology cocycles. In the interacting $A \neq 0$ case the same procedure seems to allow for only one additional supersymmetry, associated with the structure constants $f_{i j k}$ of the Lie algebra $\mathcal{G}$ considered. In the $N=1$ fermionic model, at least in the case of $\mathcal{G}=s u(n) l$ additional symmetries may be constructed directly from its cocycles. In general, these purely fermionic $\tilde{Q}$ 's depend only on the cohomology of $\mathcal{G}$ or, equivalently, on the topology of the corresponding compact group $G$. In this sense the additional supercharges may be traced to the topology of $G$; however, they may be seen to generate continuous symmetries of the system and may be obtained from Noether's theorem. In the $N=2$ case it is shown that the standard supercharges are in fact part of a series of $2 l$ conserved supercharges that can be constructed from the $l$ cocycles of $\mathcal{G}$.

Summarising, our analysis shows that the hidden symmetries appearing in $A d(G)$-invariant models are in fact supersymmetries because they stem from the existence of the $G$-invariant odd skewsymmetric tensors associated with the cohomology of $\mathcal{G}$. In this sense, these additional fermionic charges could have been introduced directly, even before having a supersymmetric model, as in the $(\mathcal{G}=s u(2))$ analysis of 19$]$ of the results of [8]. However, the fact that they square to a Casimir has more to do with the structure of the cocycles themselves [10], and hence with the structure of the generic symmetry group, than with any other considerations of $s u(2)$. In fact, the expression (29) (see also (56)), that holds for any Lie algebra $\mathcal{G}$ explains why e.g. $\left\{\tilde{Q}_{3}, \tilde{Q}_{3}\right\}$ is given by a Casimir. Also, our analysis in sec. 4 shows that the rotational symmetry of the model in [8] does not play an essential role (cf. [19]), being just a result of the fact that the fully antisymmetric tensor in 3-dimensions provides the structure constants. 
Acknowledgements. This work was partly supported by the DGICYT, Spain (\#PB 96-0756), the Junta de Castilla y León, Spain (\#C02/199) and PPARC, UK.

\section{References}

[1] G.W. Gibbons, R.H. Rietdijk and J.W. van Holten, SUSY in the sky, Nucl. Phys. B 404, 42-64 (1993)

[2] A.J. Macfarlane and A.J. Mountain, Hidden supersymmetries of particle motion in a Wu-Yang monopole field, Phys. Lett. B 373, 125-129 (1996)

[3] K. Yano, Some remarks on tensor fields and curvature, Ann. of Math. 55, 328-347 (1952)

[4] W. Dietz and R. Rüdiger, Space-times admitting Killing-Yano tensors I, Proc. R. Soc Lond. A 375, 361-378 (1981); II, ibid. A 381, 315-322 (1982)

[5] G.W. Gibbons and P.J. Ruback, The hidden symmetries of Taub-NUT and monopole scattering, Phys. Lett. B 188, 226-230 (1987); The hidden symmetries of multi-center metrics, Commun. Math. Phys. 115, 267-300 (1988)

[6] M. Tanimoto, The role of Killing-Yano tensors in supersymmetric mechanics on a curved manifold, Nucl. Phys. B 442, 549 (1995)

[7] A.J. Macfarlane, Supersymmetric quantum mechanics without dynamical bosons, Phys. Lett. B394 99-104 (1997)

[8] F. De Jonghe, A.J. Macfarlane, K. Peeters and J.W. van Holten, New supersymmetry of the monopole, Phys. Lett. B 359, 114-117 (1995)

[9] M. Plyuschay, On the nature of fermion-monopole symmetry, Phys. Lett. B 485, 187-192 (2000)

[10] J.A. de Azcárraga and A.J. Macfarlane, Compilation of identities for the antisymmetric tensors of the higher cocycles of su(n), math-ph/0006026, to appear in IJMPA 
[11] L. Brink and P. Ramond, Dirac equations light cone supersymmetry and superconformal algebras, hep-th/9908208, contributed article to Golfand's Memorial Volume, M. Shifman ed., World Scientific (1999)

[12] B. Kostant, A cubic Dirac operator and the emergence of the Euler number multiplets of representations for equal rank subgroups, Duke Math. J. 100, 447-501 (1999)

[13] J.W. van Holten, $D=1$ supergravity and spinning particles, in From field theory to quantum groups, J. Lukierski birthday volume, World Sci. (1966), p. 173

[14] J.A. de Azcárraga, A.J. Macfarlane, A.J. Mountain and J.C. Pérez Bueno, Invariant tensors for simple groups, Nucl. Phys. B510, 657-687 (1998)

[15] J.A. de Azcárraga and A.J. Macfarlane, Optimally defined RacahCasimir operators for su(n) and their eigenvalues for various classes of representations, math-ph/0006013, to appear in J. Math. Phys.

[16] J.A. de Azcárraga and A.J. Macfarlane, Fermionic realisations of simple Lie algebras and their invariant fermionic operators, hep-th/0003111, Nucl. Phys. B581, 743-760 (2000)

[17] C. Chryssomalakos, J.A. de Azcárraga, A.J. Macfarlane and J.C. Pérez Bueno, Higher order BRST and anti-BRST operators and cohomology for compact Lie algebras, hep-th/9810212, J. Math. Phys. 40, 6009-6032 (1999)

[18] A.J. Macfarlane and A.J. Mountain, Construction of supercharges for the one-dimensional supersymmetric nonlinear sigma model, JHEP 005, 9906, (1999)

[19] D. Spector, $N=0$ supersymmetry and the non-relativistic monopole, Phys. Lett. B 474, 331-335 (2000) 